\title{
$A b$ initio study of parity and time-reversal violation in laser-coolable triatomic molecules
}

\author{
Konstantin Gaul $^{1}$ and Robert Berger ${ }^{1}$ \\ ${ }^{1}$ Fachbereich Chemie, Philipps-Universität Marburg, Hans-Meerwein-Straße 4, 35032 Marburg
}

(Dated: November 15, 2018)

\begin{abstract}
Electronic structure enhancement factors of simultaneous parity and time-reversal violation $(\mathcal{P}, \mathcal{T}$ violation) caused by an electric dipole moment of the electron (eEDM) and scalar-pseudoscalar nucleon-electron current (SPNEC) interactions are reported for various metal mono-hydroxides, several of which are considered laser-coolable and promising candidates for an eEDM measurement. Electronic structure enhancements are calculated $a b$ initio within zeroth order regular approximation (ZORA) for $\mathrm{CaOH}, \mathrm{SrOH}, \mathrm{BaOH}, \mathrm{RaOH}$ and $\mathrm{YbOH}$. Scaling behavior with respect to nuclear charge numbers and the ratio of enhancement factors for both discussed sources of $\mathcal{P}, \mathcal{T}$-violation are analyzed, which are crucial to obtain stringent bounds on parameters for new physics from experiments.
\end{abstract}

High precision spectroscopy of diatomic molecules serves as powerful tool to probe high-energy scales of new physics beyond the standard model of particle physics. 1] Signatures of new physics are expected for instance from simultaneous parity $\mathcal{P}$ and time-reversal $\mathcal{T}$ violation. 2] Such a violation of fundamental symmetries can in principle result in a permanent electric dipole moment of a molecule in a vanishing electric field. With cold polar heavy molecules such as ThO currently the strictest limits on $\mathcal{P}, \mathcal{T}$-violating effects are set. 3, 4, This is due to electronic structure effects in polar heavy diatomic molecules, which strongly enhance $\mathcal{P}, \mathcal{T}$-odd effects such as an electric dipole moment of an electron (eEDM) $d_{\mathrm{e}}$ or scalar-pseudoscalar nucleon-electron current (SPNEC) interactions. [5] Refs. 6] 8 highlighted the particular situation of $\mathcal{P}, \mathcal{T}$-odd effects in the diatomic system $\mathrm{RaF}$, which was earlier identified to have the advantage of being also a molecular candidate for laser-cooling. [9] Based on simple theoretical concepts [10] it was subsequently concluded that not only diatomic, but also polyatomic molecules can be cooled with lasers. This renders such molecules promising laboratories for the study of fundamental symmetry violations. A number of molecular candidates was proposed [10] which included the particular example of $\mathrm{CaOH}$. The first successful experiment of laser-cooling of a polyatomic molecule was subsequently realised with $\mathrm{SrOH}[11$. Isaev et al. [12] suggested lasercooling of $\mathrm{RaOH}$ and its use to search for new physics. They presented also the first calculation of SPNEC interactions enhancement in a polyatomic molecule, but as of yet, no predictions on eEDM enhancement in the mentioned polyatomic candidates for a search of new physics exist.

Kozyryev et al. elucidated that laser-coolable polyatomic molecules, and in particular $\mathrm{YbOH}$, can have advantages over diatomic molecules in experimental setups and may improve sensitivity of eEDM experiments. 13. And it was pointed out in Ref. [14 that diatomic molecules are limited in the sensitivity of a simultaneous determination of $d_{\mathrm{e}}$ as well as the coupling constant of SPNEC interactions $k_{\mathrm{s}}$ when one analyses the scaling behaviour of the enhancement factors with respect to the charge of the heavy nucleus.

To provide these enhancement factors for upcoming experiments on triatomic molecules, we present in this letter predictions of $W_{\mathrm{d}}$ and $W_{\mathrm{s}}$, the electronic structure enhancement factors of an eEDM and SPNEC interactions, respectively, in the laser-coolable polyatomic molecules $\mathrm{CaOH}, \mathrm{SrOH}, \mathrm{RaOH}$ and $\mathrm{YbOH}$, as well as for $\mathrm{BaOH}$, which is isoelectronic to $\mathrm{BaF}$, a promising candidate for the first detection of molecular parity-violation. 15. We compare herein also the ratio $W_{\mathrm{d}} / W_{\mathrm{s}}$ to those obtained for diatomic molecules in order to gauge the advantage of polyatomic over diatomic molecules with respect to electronic structure enhancement effects.

The metal hydroxides $(\mathrm{MOH})$ studied herein are linear molecules and expected to have a ${ }^{2} \Sigma_{1 / 2}$-ground state. Thus the effective $\mathcal{P}, \mathcal{T}$-odd spin-rotational Hamiltonian is the same as for diatomic molecules studied in Ref. [14, if one neglects contributions of the light nuclei, namely:

$$
H_{\mathrm{sr}}=\left(k_{\mathrm{s}} W_{\mathrm{s}}+d_{\mathrm{e}} W_{\mathrm{d}}\right) \Omega,
$$

where $\Omega=\vec{J}_{\mathrm{e}} \cdot \vec{\lambda}$ is the projection of the reduced total electronic angular momentum $\vec{J}_{\mathrm{e}}$ on the molecular axis, defined by the unit vector $\vec{\lambda}$ pointing from the heavy nucleus to the $\mathrm{OH}$-group. $k_{\mathrm{s}}$ is the $\mathcal{P}, \mathcal{T}$-odd scalar-pseudoscalar nucleon-electron current interaction constant and $d_{\mathrm{e}}$ is the eEDM. The $\mathcal{P}, \mathcal{T}$-odd electronic structure parameters are defined by

$$
W_{\mathrm{s}}=\frac{\left\langle\Psi\left|\hat{H}_{\mathrm{s}}\right| \Psi\right\rangle}{k_{\mathrm{s}} \Omega} \text { and } W_{\mathrm{d}}=\frac{\left\langle\Psi\left|\hat{H}_{\mathrm{d}}\right| \Psi\right\rangle}{d_{\mathrm{e}} \Omega},
$$

where $\Psi$ is the electronic wave function. The molecular $\mathcal{P}, \mathcal{T}$-odd Hamiltonians 2, 16, 17 .

$$
\begin{aligned}
& \hat{H}_{\mathrm{s}}=1 k_{\mathrm{s}} \frac{G_{\mathrm{F}}}{\sqrt{2}} \sum_{i=1}^{N_{\text {elec }}} \sum_{A=1}^{N_{\text {nuc }}} \rho_{A}\left(\vec{r}_{i}\right) Z_{A} \gamma^{0} \gamma^{5} \\
& \hat{H}_{\mathrm{d}}=\frac{21 c d_{\mathrm{e}}}{\hbar e} \sum_{i=1}^{N_{\text {elec }}} \gamma^{0} \gamma^{5} \hat{\vec{p}}_{i}^{2} .
\end{aligned}
$$


were implemented and evaluated in a quasi-relativistic framework within zeroth order regular approximation (ZORA):[14, 18]

$$
\begin{aligned}
& \hat{H}_{\mathrm{s}}^{\mathrm{ZORA}}=1 \sum_{i=1}^{N_{\text {elec }}} \sum_{A=1}^{N_{\text {nuc }}} Z_{A}\left[\rho_{A}\left(\vec{r}_{i}\right) \tilde{\omega}_{\mathrm{s}}\left(\vec{r}_{i}\right), \overrightarrow{\boldsymbol{\sigma}} \cdot \hat{\vec{p}}_{i}\right]_{-} \\
& \hat{H}_{\mathrm{d}}^{\mathrm{ZORA}}=1 \sum_{i=1}^{N_{\text {elec }}} \hat{\vec{p}}_{i}^{2} \tilde{\omega}_{\mathrm{d}}\left(\vec{r}_{i}\right)\left(\overrightarrow{\boldsymbol{\sigma}} \cdot \hat{\vec{p}}_{i}\right)-\left(\overrightarrow{\boldsymbol{\sigma}} \cdot \hat{\vec{p}}_{i}\right) \tilde{\omega}_{\mathrm{d}}\left(\vec{r}_{i}\right) \hat{\vec{p}}_{i}^{2}
\end{aligned}
$$

Here $\rho_{A}$ is the normalized nuclear charge density distribution of nucleus $A$ with charge number $Z_{A}, \vec{r}_{i}$ is the position vector of electron $i, G_{\mathrm{F}}=2.22249 \times 10^{-14} E_{\mathrm{h}} a_{0}^{3}$ is Fermi's weak coupling constant, $1=\sqrt{-1}$ is the imaginary unit, $\hat{\vec{p}}$ is the linear momentum operator, $\vec{\sigma}$ is the vector of the Pauli spin matrices, $[A, B]_{-}=A B-B A$ is the commutator and the modified ZORA factors are defined as

$$
\begin{aligned}
\tilde{\omega}_{\mathrm{s}}\left(\vec{r}_{i}\right) & =\frac{G_{\mathrm{F}} k_{\mathrm{s}} c}{\sqrt{2}\left(2 m_{\mathrm{e}} c^{2}-\tilde{V}\left(\vec{r}_{i}\right)\right)}, \\
\tilde{\omega}_{\mathrm{d}}\left(\vec{r}_{i}\right) & =\frac{2 d_{\mathrm{e}} c^{2}}{2 e \hbar m_{\mathrm{e}} c^{2}-e \hbar \tilde{V}\left(\vec{r}_{i}\right)} .
\end{aligned}
$$

with the model potential $\tilde{V}$ introduced by van Wüllen[19], which is used to alleviate the gauge dependence of ZORA. Here $c$ is the speed of light in vacuum, $\hbar=\frac{h}{2 \pi}$ is the reduced Planck constant and $m_{\mathrm{e}}$ is the mass of the electron.

On the technical side, the quasi-relativistic twocomponent calculations reported herein are performed within ZORA at the level of complex generalized Hartree-Fock (cGHF) or Kohn-Sham (cGKS) with a modified version [20 23] of the quantum chemistry program package Turbomole 24]. For details on our implementation of $\mathcal{P}, \mathcal{T}$-odd properties within this ZORA framework see Refs. [14, 18, 25]. For Kohn-Sham (KS)-density functional theory (DFT) calculations the hybrid Becke three parameter exchange functional and Lee, Yang and Parr correlation functional (B3LYP) 2629] was employed. For all calculations a basis set of $37 \mathrm{~s}, 34 \mathrm{p}, 14 \mathrm{~d}$ and $9 \mathrm{f}$ uncontracted Gaussian functions with the exponential coefficients $\alpha_{i}$ composed as an even-tempered series as $\alpha_{i}=a \cdot b^{N-i} ; i=1, \ldots, N$, with $b=2$ for s- and p-function and with $b=(5 / 2)^{1 / 25} \times$ $10^{2 / 5} \approx 2.6$ for $\mathrm{d}$ - and f-functions was used for the electro-positive atom (for details see Supplementary Material). The $\mathrm{N}, \mathrm{F}$ and $\mathrm{O}$ atoms were represented with a decontracted atomic natural orbital (ANO) basis set of triple- $\zeta$ quality 30 and for $\mathrm{H}$ the s,p-subset of a decontracted correlation-consistent basis of quadruple- $\zeta$ quality 31] was used. The ZORA-model potential $\tilde{V}(\vec{r})$ was employed with additional damping 32 as proposed by van Wüllen[19]. For two-component wave functions and properties a finite nucleus was used, described by a normalized spherical Gaussian nuclear density distri- bution $\rho_{A}(\vec{r})=\rho_{0} \mathrm{e}^{-\frac{3}{2 \zeta_{A}} \vec{r}^{2}}$. The root mean square radius $\zeta_{A}$ of nucleus $A$ was used as suggested by Visscher and Dyall, 33 , where the mass numbers $A$ are ${ }^{40} \mathrm{Ca}$, ${ }^{137} \mathrm{Ba},{ }^{173} \mathrm{Yb},{ }^{226} \mathrm{Ra}$. The nuclear equilibrium distances were obtained at the levels of GHF-ZORA and GKSZORA/B3LYP, respectively. For calculations of energy gradients at the DFT level the nucleus was approximated as a point charge. The molecular structure parameters obtained are summarized in Table 1 on page 4.

Our results for $W_{\mathrm{d}}$ and $W_{\mathrm{s}}$ are presented together with angular momentum quantum numbers $\Omega$ in Table II] on page 5. All $\Omega$ values are close to the expected value ${ }^{1} / 2$. Minor numeric deviations from ${ }^{1} / 2$ are due to slight numeric deviations of the computed equilibrium structure from linearity and, caused by this, an imperfect alignment on the $z$-axis.

Values calculated for $W_{\mathrm{d}}$ and $W_{\mathrm{s}}$ on the DFT level for group 2 hydroxides differ only slightly from those obtained with GHF, which is in agreement with previous studies of $\mathcal{P}, \mathcal{T}$-violation in group 2 compounds. 14] The larger deviation for $\mathrm{YbOH}$ shows that electron correlation effects may be more important for this f-block compound. However, previous comparisons of our method with coupled-cluster four-component calculations shows that the accuracy of the present approach can be estimated to be on the order of $20 \%$, which is fully sufficient for the present purpose.

We find that compared to $\mathcal{P}, \mathcal{T}$-odd enhancement in metal fluorides, calculated in Ref. [14, the values for the corresponding hydroxides are slightly larger in magnitude, but all in all differences are very small, below $5 \%$. Considering possible improvements of the experimental setup with polyatomic molecules as described in Ref. [13], experiments with laser-coolable $\mathrm{RaOH}$ or $\mathrm{YbOH}$ as promising candidates for an improvement of current limits on the eEDM consequently would benefit mainly from full polarisation of the molecule and the existence of internal co-magnetometer states, but not from improved electronic enhancement factors. The potential of the latter in polyatomic molecules is thus yet to be explored.

For further insight the scaling with nuclear charge $Z$ is studied. For this purpose non-linear relativistic enhancement is separated as described in Ref. [14 using relativistic enhancement factors known from atomic considerations 34 36 $R_{\mathrm{s}}=R(Z, A) f(Z) \frac{\gamma+1}{2}$ and $R_{\mathrm{d}}=\frac{1}{\gamma^{4}}$ with $f(Z)=\frac{1-0.56 \alpha^{2} Z^{2}}{\left(1-0.283 \alpha^{2} Z^{2}\right)^{2}}$ and $R(Z, A)=$ $\frac{4}{\Gamma^{2}(2 \gamma+1)}\left(2 Z r_{\text {nuc }} / a_{0}\right)^{2 \gamma-2}$. Here $\alpha$ is the fine structure constant, $a_{0}$ is Bohr's radius and $r_{\text {nuc }} \approx 1.2 \mathrm{fm} \cdot A^{1 / 3}$. A double logarithmic plot for cGHF and cGKS results together with a linear fit is presented in Figure 1 on page 5 . The $Z$-dependence of $Z^{2.83}$ for $W_{\mathrm{s}}$ (cGKS) is similar to that reported for group 2 fluorides in Ref. [14] of $Z^{2.79}$ for $W_{\mathrm{s}}(\mathrm{cGKS})$, whereas $W_{\mathrm{d}}$ scales steeper for $\mathrm{MOH}\left(Z^{2.77}\right)$ than for group 2 fluorides (Ref. [14: $Z^{2.57}$ ).

As discussed in detail in Ref. 14 the ratio $W_{\mathrm{d}} / W_{\mathrm{s}}$ of two different experiments determines if the experiments 
are complementary or redundant for a parallel determination of $k_{\mathrm{s}}$ and $d_{\mathrm{e}}$. In Table III on page 5 the ratios $W_{\mathrm{d}} / W_{\mathrm{s}}$ are compared to those of corresponding fluorides determined in Ref. 14.

The values show that the metal hydroxides fit perfectly in the model developed in Ref. 14. Hence there is in this sense no immediate advantage of using a metal hydroxide instead of a fluoride. With respect to the coverage region in the parameter space of $k_{\mathrm{s}}$ and $d_{\mathrm{e}}$, however, an experiment with $\mathrm{MOH}$ would be able to reduce the size of the coverage region due to the expected smaller experimental uncertainties. Experiments with the corresponding MF compounds would become redundant as essentially the same information is measured.

In this letter we reported the calculation of enhancements of an electric dipole moment of the electron in simple polyatomic molecules. Our calculations show that there is no considerable difference for enhancement factors between fluorides and hydroxides. This is also true for the ratio $W_{\mathrm{d}} / W_{\mathrm{s}}$. Thus there is no advantage in using $\mathrm{MOH}$ alongside MF in experiment as both experiments yield the same information on the parameter space of $d_{\mathrm{e}}$ and $k_{\mathrm{s}}$. In order to see distinct differences to diatomic molecules it may be necessary to study more complex and non-linear polyatomic molecules. However, together with the experimental benefits of polyatomic molecules described in Ref. [13] the herein studied molecules are promising candidates for an improvement of current limits on $\mathcal{P}, \mathcal{T}$-violating effects.

\section{ACKNOWLEDGMENTS}

We thank Timur Isaev for inspiring discussions. Computer time provided by the center for scientific computing (CSC) Frankfurt is gratefully acknowledged.
[1] D. DeMille, Physics Today 68, 34 (2015), https://doi.org/10.1063/PT.3.3020

[2] I. B. Khriplovich and S. K. Lamoreaux, CP Violation without Strangeness (Springer, Berlin, 1997).

[3] J. Baron, W. C. Campbell, D. DeMille, J. M. Doyle, G. Gabrielse, Y. V. Gurevich, P. W. Hess, N. R. Hutzler, E. Kirilov, I. Kozyryev, B. R. O'Leary, C. D. Panda, M. F. Parsons, E. S. Petrik, B. Spaun, A. C. Vutha, and A. D. West, Science 343, 269 (2014), arXiv:1310.7534.

[4] V. Andreev, D. G. Ang, D. DeMille, J. M. Doyle, G. Gabrielse, J. Haefner, N. R. Hutzler, Z. Lasner, C. Meisenhelder, B. R. O'Leary, C. D. Panda, A. D. West, E. P. West, X. Wu, and A. C. M. E. Collaboration, Nature 562, 355 (2018).

[5] J. S. M. Ginges and V. V. Flambaum, Phys. Rep. 397, $63(2004)$

[6] T. A. Isaev and R. Berger, ArXiv e-prints (2013), arXiv:1302.5682 [physics.chem-ph]

[7] A. D. Kudashov, A. N. Petrov, L. V. Skripnikov, N. S. Mosyagin, T. A. Isaev, R. Berger, and A. V. Titov, Phys. Rev. A 90, 052513 (2014).

[8] S. Sasmal, H. Pathak, M. K. Nayak, N. Vaval, and S. Pal, Phys. Rev. A 93, 062506 (2016).

[9] T. A. Isaev, S. Hoekstra, and R. Berger, Phys. Rev. A 82, $052521(2010)$.

[10] T. A. Isaev and R. Berger, Phys. Rev. Lett. 116, 063006 (2016)

[11] I. Kozyryev, L. Baum, K. Matsuda, B. L. Augenbraun, L. Anderegg, A. P. Sedlack, and J. M. Doyle, Phys. Rev. Lett. 118, 173201 (2017).

[12] T. A. Isaev, A. V. Zaitsevskii, and E. Eliav, J. Phys. B 50, 225101 (2017).

[13] I. Kozyryev and N. R. Hutzler, Phys. Rev. Lett. 119, $133002(2017)$

[14] K. Gaul, S. Marquardt, T. A. Isaev, and R. Berger, ArXiv e-prints (2018), arXiv:1805.05494 [physics.chemph]

[15] E. Altuntaş, J. Ammon, S. B. Cahn, and D. DeMille, Phys. Rev. Lett. 120, 142501 (2018).
[16] E. Salpeter, Phys. Rev. 112, 1642 (1958).

[17] A. Mårtensson-Pendrill and P. Öster, Phys. Scr. 36, 444 (1987)

[18] K. Gaul and R. Berger, J. Chem. Phys. 147, 014109 (2017)

[19] C. van Wüllen, J. Chem. Phys. 109, 392 (1998)

[20] R. Berger, N. Langermann, and C. van Wüllen, Phys. Rev. A 71, 042105 (2005).

[21] R. Berger and C. van Wüllen, J. Chem. Phys. 122, 134316 (2005)

[22] S. Nahrwold and R. Berger, J. Chem. Phys. 130, 214101 (2009)

[23] C. van Wüllen, Z. Phys. Chem 224, 413 (2010).

[24] R. Ahlrichs, M. Bär, M. Häser, H. Horn, and C. Kölmel, Chem. Phys. Lett. 162, 165 (1989)

[25] T. A. Isaev and R. Berger, Phys. Rev. A 86, 062515 (2012)

[26] P. J. Stephens, F. J. Devlin, C. F. Chabalowski, and M. J. Frisch, J. Phys. Chem. 98, 11623 (1994).

[27] S. H. Vosko, L. Wilk, and M. Nuisar, Can. J. Phys. 58, $1200(1980)$,

[28] A. D. Becke, Phys. Rev. A 38, 3098 (1988).

[29] C. Lee, W. Yang, and R. G. Parr, Phys. Rev. B 37, 785 (1988)

[30] B. O. Roos, R. Lindh, P. k. Malmqvist, V. Veryazov, and P. O. Widmark, J. Phys. Chem. A 108, 2851 (2004).

[31] T. H. Dunning, Jr., J. Chem. Phys. 90, 1007 (1989).

[32] W. Liu, C. van Wüllen, F. Wang, and L. Li, J. Chem. Phys. 116, 3626 (2002).

[33] L. Visscher and K. G. Dyall, At. Data Nucl. Data Tables 67, 207 (1997).

[34] V. A. Dzuba, V. V. Flambaum, and C. Harabati, Phys. Rev. A 84, 052108 (2011).

[35] E. Fermi and E. Segrè, Mem. Acad. d'Italia 4, 131 (1933).

[36] E. Fermi and E. Segrè, Z. Phys. 82, 729 (1933) 
Table I. Molecular structure parameters calculated with in a quasi-relativistic ZORA approach at the cGHF and cGKS/B3LYP level for radical metalhydroxides $\mathrm{MOH}$ with $\mathrm{M}=\mathrm{Ca}, \mathrm{Sr}, \mathrm{Ba}, \mathrm{Ra}, \mathrm{Yb}$.

\begin{tabular}{|c|c|c|c|c|c|c|}
\hline M & \multicolumn{2}{|c|}{$r(\mathrm{M}-\mathrm{O}) / \AA$} & \multicolumn{2}{|c|}{$r(\mathrm{O}-\mathrm{H}) / \AA$} & \multicolumn{2}{|c|}{$\varangle(\mathrm{M}-\mathrm{O}-\mathrm{H}) /^{\circ}$} \\
\hline $\mathrm{Ca}$ & 2.006 & 1.972 & 0.932 & 0.954 & 179.91 & 179.70 \\
\hline $\mathrm{Sr}$ & 2.134 & 2.110 & 0.933 & 0.955 & 179.99 & 179.93 \\
\hline $\mathrm{Ba}$ & 2.239 & 2.207 & 0.935 & 0.956 & 179.93 & 179.95 \\
\hline $\mathrm{Ra}$ & 2.315 & 2.289 & 0.935 & 0.956 & 179.93 & 179.93 \\
\hline $\mathrm{Yb}$ & 2.083 & 2.002 & 0.933 & 0.953 & 179.92 & 179.92 \\
\hline
\end{tabular}


Table II. Angular momentum and $\mathcal{P}, \mathcal{T}$-violating properties of hydroxide radicals calculated $a b$ initio within a quasi-relativistic two-component ZORA approach at the cGHF and cGKS/B3LYP level. Dev. refers to the relative deviation between cGHF and cGKS results.

\begin{tabular}{|c|c|c|c|c|c|c|c|c|c|}
\hline \multirow[t]{2}{*}{ Molecule } & \multirow[t]{2}{*}{$Z$} & \multicolumn{2}{|c|}{$\Omega^{*}$} & \multicolumn{3}{|c|}{$W_{\mathrm{s}} \frac{1}{h \cdot \mathrm{Hz}}$} & \multicolumn{3}{|c|}{$W_{\mathrm{d}} \frac{e \cdot \mathrm{cm}}{10^{24} \cdot h \cdot \mathrm{Hz}}$} \\
\hline & & $\mathrm{cGHF}$ & cGKS & cGHF & cGKS & $\overline{\text { Dev. }}$ & cGHF & cGKS & Dev. \\
\hline $\mathrm{CaOH}$ & 20 & -0.494 & -0.499 & $-2.18 \times 10^{2}$ & $-2.14 \times 10^{2}$ & $2 \%$ & $-1.44 \times 10^{-1}$ & $-1.41 \times 10^{-1}$ & $2 \%$ \\
\hline $\mathrm{SrOH}$ & 38 & -0.500 & -0.500 & $-2.00 \times 10^{3}$ & $-1.97 \times 10^{3}$ & $1 \%$ & -1.04 & -1.03 & $1 \%$ \\
\hline $\mathrm{BaOH}$ & 56 & 0.483 & 0.483 & $-8.79 \times 10^{3}$ & $-7.91 \times 10^{3}$ & $10 \%$ & -3.32 & -2.98 & $10 \%$ \\
\hline $\mathrm{RaOH}$ & 88 & 0.494 & 0.471 & $-1.53 \times 10^{5}$ & $-1.41 \times 10^{5}$ & $8 \%$ & $-2.75 \times 10^{1}$ & $-2.53 \times 10^{1}$ & $8 \%$ \\
\hline $\mathrm{YbOH}$ & 70 & -0.500 & -0.495 & $-4.12 \times 10^{4}$ & $-3.08 \times 10^{4}$ & $25 \%$ & $-1.14 \times 10^{1}$ & -8.54 & $25 \%$ \\
\hline
\end{tabular}

${ }^{*}$ The absolute sign of $\Omega$ is arbitrary. However, relative to the sign of the effective electric field $W_{\mathrm{d}} \Omega$ it is always such that $\operatorname{sgn}\left(W_{\mathrm{d}}\right)=-1$.
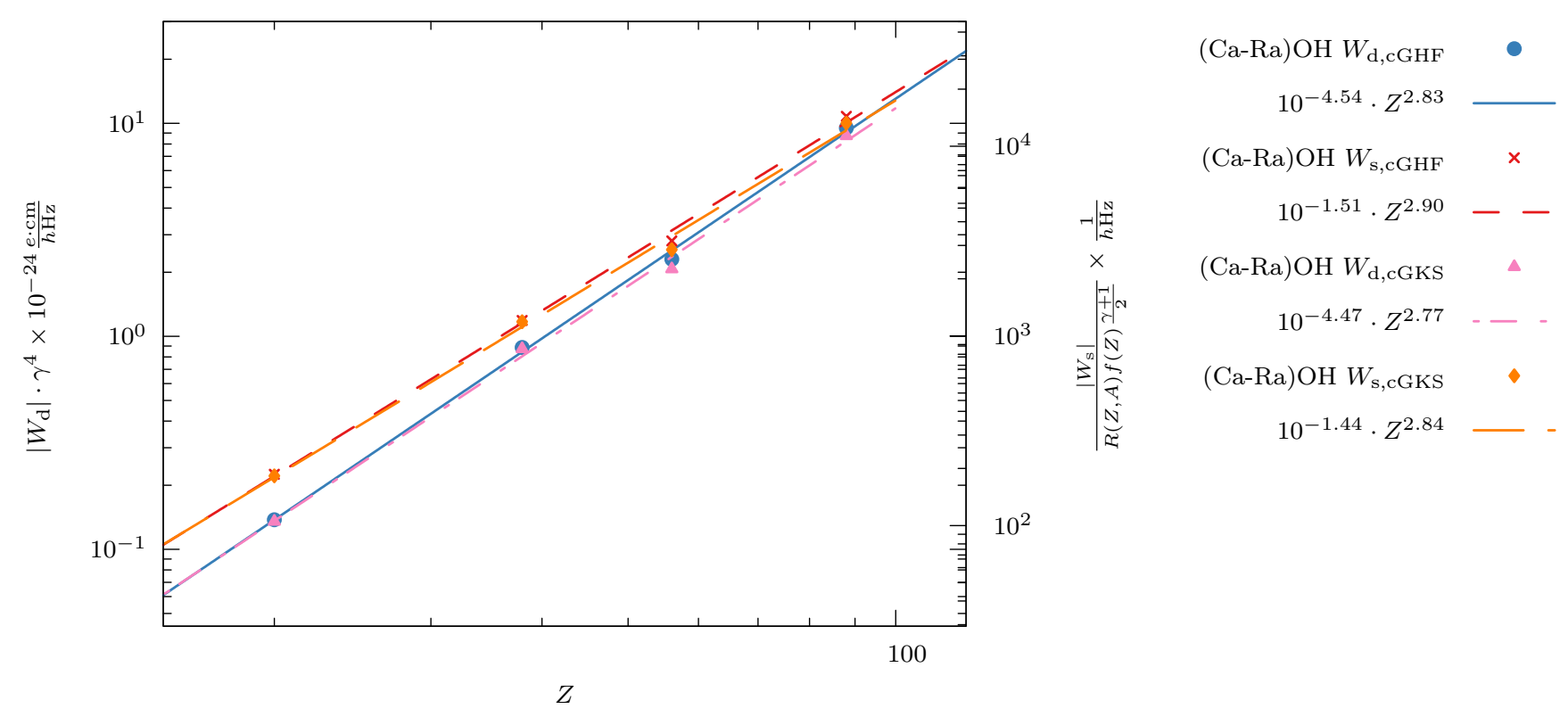

Figure 1. Scaling of $\log _{10}\left\{\left|W_{\mathrm{d}}\right| \gamma^{4} \times 10^{-24} \frac{e \cdot \mathrm{cm}}{h \mathrm{~Hz}}\right\}$ and $\log _{10}\left\{\frac{\left|W_{\mathrm{s}}\right|}{R(Z, A) f(Z) \frac{\gamma+1}{2}} \times \frac{1}{h \mathrm{~Hz}}\right\}$ with $\log _{10}\{Z\}$ for group 2 fluorides (Ca-Ra)F at the level of GKS-ZORA/B3LYP and GHF-ZORA.

Table III. $\mathcal{P}, \mathcal{T}$-odd ratios $W_{\mathrm{d}} / W_{\mathrm{s}}$ of hydroxide radicals $\mathrm{MOH}$ calculated within a quasi-relativistic two-component ZORA approach at the cGKS/B3LYP level in comparison to ratios of corresponding fluoride radicals MF calculated in Ref. 14

\begin{tabular}{lll}
\hline $\mathrm{M}$ & \multicolumn{2}{c}{$W_{\mathrm{d}} / W_{\mathrm{s}} \times 10^{-20} e \cdot \mathrm{cm}$} \\
\cline { 2 - 3 } & $\mathrm{MOH}$ & $\mathrm{MF}$ \\
\hline $\mathrm{Ca}$ & 6.60 & 6.62 \\
$\mathrm{Sr}$ & 5.22 & 5.17 \\
$\mathrm{Ba}$ & 3.77 & 3.78 \\
$\mathrm{Ra}$ & 1.79 & 1.79 \\
$\mathrm{Yb}$ & 2.77 & 2.76 \\
\hline
\end{tabular}

